\title{
Association of circulating oxidized LDL-C/LDL-C ratio with severity of coronary atherosclerosis and other emerging biomarkers of cardiovascular disease in patients with type 2 diabetes
}

Bo Feng ( $\sim$ fengbodfyy@tongji.edu.cn )

Original investigation

Keywords: OxLDL, Atherosclerosis cardiovascular diseases, biomarkers, diabetes mellitus

Posted Date: October 12th, 2021

DOI: https://doi.org/10.21203/rs.3.rs-962747/v1

License: (a) (1) This work is licensed under a Creative Commons Attribution 4.0 International License. Read Full License 


\section{Abstract \\ Background}

Atherosclerosis cardiovascular diseases (ASCVD) are the leading cause of mortality and morbidity in patients with diabetes. But there are still no simpler and more effective biomarkers which can predict the severity of coronary atherosclerosis in diabetic patients. Oxidized low-density lipoprotein (oxLDL) is a novel CVD biomarker that also increase in diabetic patients. The aim of present study was to evaluate the associations of circulating oxLDL/LDL-C ratio with severity of coronary atherosclerosis and other emerging biomarkers of cardiovascular disease such as fibroblast growth factor 21 (FGF21), interleukin 33 (IL33) and vascular cell adhesion molecule-1 (VCAM-1) in patients with type 2 diabetes.

\section{Methods}

152 patients with type 2 diabetes were recruited in our study. Plasma levels in oxLDL, FGF21, IL33 and VCAM-1 were measured by ELISA. oxLDL/LDL-C ratio was calculated. Coronary computed tomographic angiography (CCTA) was performed in all patients. Patients were allocated into four groups according to CCTA findings and Gensini score: normal, mild, moderate and severe coronary atherosclerosis group. Association between the oxLDL/LDL-C ratio and the severity of coronary atherosclerosis were evaluated using logistic regression models and receiver operator characteristic (ROC) curve analyses.

\section{Results}

Correlation analysis showed that oxLDL/LDL-C ratio was positively associated with severity of coronary atherosclerosis $(p<0.05)$. After adjusted for age, duration of diabetes, the positive correlation between severity of coronary atherosclerosis and oxLDL/LDL-C ratio still existed (OR 2.03, 95\% Cl 1.31-3.14, p < 0.01). OxLDL/LDL-C ratio in severe group was significantly higher than which in the other three groups respectively ( $p<0.001)$. The cut-off value of oxLDL/LDL-C ratio in predicting severe coronary atherosclerosis was more than 0.091 with specificity of $66.5 \%$ and sensitivity of $75 \%$. Only IL33 correlated positively with oxLDL/LDL-C ratio $(r=0.274, p<0.01)$. However, VCAM- 1 had a similar trend with oxLDL/LDL-C ratio in these four groups.

\section{Conclusions}

OxLDL/LDL-C ratio is considered as a potential biomarker in diabetic patients for early recognition and intervention of severe coronary atherosclerosis, and will be more effective if tested IL33 and VCAM-1 at the same time.

\section{Background}


The prevalence of diabetes mellitus is increasing significantly worldwide, especially around $10.4 \%$ in China ${ }^{[1]}$. Patients with diabetes are at higher risk of microvascular and macrovascular complications. Cardiovascular diseases (CVD) are the primary cause of mortality and morbidity in diabetes. It is unquestionable that diabetes is the most important risk factor for cardiovascular disease ${ }^{[2]}$.The arterial involvement is characterized by the development of accelerated atherosclerosis, which leads to either coronary artery disease, cerebrovascular accidents, or peripheral vascular disease ${ }^{[3]}$. Early detection and management of cardiovascular risk factors are important in the prevention of incident CVD events in patients with diabetes. However, it is difficult to identify diabetic individuals with higher CVD risk for early intervention, highlighting the need to identify additional biomarkers for CVD risk screening and severity evaluation.

Low-density lipoprotein cholesterol (LDL-C) has long been identified as a circulating biomarker reflecting general cardiovascular risk, with a reduction in serum levels used clinically as a treatment target. However, there is no sufficient clinical evidence that LDL-C level can predict the severity of coronary artery disease to date. Atherosclerosis is a complex process, and there is still scope for more specific biomarkers with pathological relevance to improve the clinical risk prediction of cardiovascular events especially in patients with diabetes ${ }^{[4]}$. Native LDL-C, however, cannot induce cholesterol accumulation in monocytes/macrophages which increase, rather, is due to the uptake of one or more modified forms of LDL-C ${ }^{[5]}$. The major modification that can induce cholesterol accumulation is oxidized LDL-C ${ }^{[6]}$. It is hypothesized that under conditions of oxidative stress, oxLDL formation predominantly occurs within the extracellular space of the vascular wall. Patients with diabetes exhibit a decreased plasma antioxidant activity as well as increased levels of lipid hydroperoxides and F2-isoprotanes. The latter two are recognized in vivo markers of oxidative stress ${ }^{[7]}$. Hyperglycemia-induced oxidative stress modifies LDL-C into an oxLDL form ${ }^{[8]}$. Moreover, elevated oxLDL titres may prove to be more causally associated with cardiovascular events than LDL-C, due to its central role in atherosclerotic plaque biology, and thus may have potential for improving risk prediction beyond LDL-C in patients with diabetes. The present observations suggest that oxLDL and ox-LDL/LDL-C are better biomarkers than popular lipid profile [total cholesterol (TC), LDL-C, high density lipoprotein-cholesterol (HDL-C)] for discriminating between patients with CAD and healthy subjects ${ }^{[9]}$.

There has been evidences that oxLDL is increased in subclinical atherosclerosis ${ }^{[10]}$ and is often a stronger predictor of acute coronary artery disease (CAD) than standard lipid measures or other conventional risk factors ${ }^{[11]}$. OxLDL levels are reportedly able to distinguish patients with CAD from healthy cohorts ${ }^{[12]}$, and serve as a predictor of future myocardial infarction in patients with unstable CAD ${ }^{[13]}$. OxLDL level is also associated with type 2 diabetes mellitus (T2DM) ${ }^{[14]}$. Previous studies have examined the ratios of oxLDL/LDL-C and demonstrated that this metric is more informative than oxLDL alone. Data from these study revealed that the correlationship between CAD and relative degree of LDL-C oxidation is stronger than with the level of oxidized LDL-C in vivo ${ }^{[15-17]}$. Most of evidences suggeste that oxLDL or oxLDL/LDL-C ratio can predict future cardiovascular events. However it remains unclear 
whether it can predict the severity of coronary atherosclerosis. The association between circulating oxLDL and the severity of coronary atherosclerosis yielded conflicting results. In several studies, oxLDL levels were not significantly related to the extent of coronary heart disease (CHD) ${ }^{[18-19]}$, but in other studies the association was not found ${ }^{[20]}$. Especially there was no report about the relationship in patients with diabetes. Since cardiovascular events are irreversible, it will be more meaningful to recognize severe coronary atherosclerosis earlier through some effective biomarkers.

Since studies have demonstrated that the severity of CAD assessed by Gensini score is associated with increased cardiovascular events ${ }^{[21]}$. The present study aimed to assess the relationship between oxLDL/LDL-C ratio with severity of coronary atherosclerosis through Gensini scores calculated on the basis of coronary computed tomographic angiography (CCTA) in patients with diabetes. Recent advances in CT technology improve significantly the diagnostic accuracy of CCTA, which leads to substantial increases in its non-invasive use for patients with suspected CAD [22]. It was reported that the presence and severity of CAD visualized by CCTA predict death or MI across different large ethnicities [23]. Current evidences show that the calculation of SYNTAX score derived from CCTA is accurate with respect to the one derived from ICA assessment ${ }^{[24]}$. Many studies calculated Gensini score derived from CCTA and proved the accuracy and its impacts ${ }^{[25-27]}$.

Moreover in present study we detected some emerging biomarkers such as FGF21 and IL33, which were reported to be related with diabetes and CAD in some contradictory results, and conventional biomarker such as VCAM-1. FGF21 is a multifunctional protein with major secretion and expression in the adipose tissues and liver with control over energy, glucose, and lipid metabolism [28]. There are higher levels in FGF21 in patients with AS, according to current preclinical and clinical reports ${ }^{[29-30]}$. IL-33 exerts its cellular functions by binding a receptor complex composed of suppression of tumorigenity (ST2L) and IL$1 \mathrm{R}$ accessory protein ${ }^{[31]}$. According to current knowledge, IL-33 seems to be released during necrotic cell death, which is thought to be associated with tissue damage. For these properties, IL-33 was proposed to act as an "alarmin" ${ }^{[32]}$. VCAM-1 is the transmembrane glycoproteins that are responsible for transmigrating leukocytes into the vascular intima. Elevated expression and activity of VCAM- 1 is indicative of inflammation, endothelial dysfunction, and atherosclerosis ${ }^{[33]}$. The present study tried to investigate the relationship of these biomarkers with oxLDL/LDL-C ratio and the severity of diabetic coronary atherosclerosis.

\section{Methods}

\section{Study participants}

A total of 152 patients with type 2 diabetes at the Shanghai East Hospital, Tongji university, were included in our study. Patients were diagnosed according to the 1999 World Health Organization criteria. Patients with type 1 diabetes mellitus or specific types of diabetes mellitus, acute complications of diabetes, liver or renal dysfunction, serious cardiac arrhythmias or acute cardiac insufficiency, diabetic foot ulcers and 
history of cerebral infarction, tumor and psychosis were excluded. Written informed consents were obtained from all participants. The study was approved by the Human Research and Ethics Committee of Shanghai East Hospital and adhered to the tenets of the Declaration of Helsinki. The study was registered in the Chinese clinical trial registry (ChiCTR-2100047148).

\section{Data collection and physical assessment}

Information on sex, age, weight, height, duration of diabetes, and history of smoking, coronary artery disease, hypertension and cerebral apoplexy were obtained using a questionnaire. The body mass index (BMI) was calculated as the weight $(\mathrm{kg})$ divided by the square of height $(\mathrm{m})$. Blood pressure was measured using a sphygmomanometer during a physical examination.

\section{Biochemical assessment}

Venous blood samples were collected from all patients after overnight fasting. The levels of TC, LDL-C, HDL-C, triglycerides (TG), fasting plasma glucose (FPG), glycosylated hemoglobin ( $\mathrm{HbA1c}$ ) and serum creatinine were measured by suitable laboratory techniques including HPLC for HbA1c. Serum samples stored at $-80^{\circ} \mathrm{C}$ were used for the determination of oxLDL levels using ELISA assays kit supplied by Mercodia (Uppsala, Sweden). Serum FGF21, IL33 and VCAM-1 were determined by ELISA kits supplied by Abcam (Cambridge, MA, USA).

\section{Coronary computed tomographic angiography and Gensini score calculation}

A 64-multi-detector computed tomography scanner (Aquilion ONE TSX-301C, TOSHIBA囚was used. Imaging range was as follows: 1 inch above the left main ostium down to 1 inch below the bottom of the heart. Cardiac data were reconstructed from 5 to $95 \%$ of $R-R$ interval and with $10 \%$ intervals. Coronary vessels were analyzed. Volume renderings and curved multiplanar reformations were reported. Imaging of coronary arteries was established with the use of 30-40 contiguous $3 \mathrm{~mm}$ slices (mid-diastole) with ECG triggering and breath holding for $15 \mathrm{~s}$.

In this study, severity of coronary atherosclerosis was assessed by Gensini score, which is based on the percentage of luminal narrowing. The Gensini score was computed by assessing the severity score of each coronary stenosis according to the degree of luminal narrowing and its geographic importance. Reduction in the lumen diameter and appearance of concentric lesion or eccentric plaques were evaluated (reduction of $25 \%, 50 \%, 75 \%, 90 \%, 99 \%$, and complete occlusion were given Gensini scores of 1 , $2,4,8,16$, and 32, respectively). Each vascular segment was assigned a multiplier in accordance with the functional significance of the myocardial area supplied by that segment: the left main coronary artery $\times 5$; the proximal segment of left anterior descending artery $(L A D) \times 2.5$; the proximal segment circumflex artery $\times 2.5$; the mid segment of the LAD $\times 1.5$; the right coronary artery, the distal segment of LAD, the posterolateral artery, and the obtuse marginal artery $\times 1$; and others $\times 0.5^{[34]}$. All patients were allocated into four groups according to CCTA findings and Gensini score: normal group (score=0), mild coronary 
atherosclerosis group ( $0<$ scores $\leq 3)$, moderate coronary atherosclerosis group $(3.01 \leq$ scores $\leq 32.67)$ and severe coronary atherosclerosis group (32.68 $\leq$ scores $\leq 180)$.

\section{Statistical methods}

For statistical analysis, SPSS 21.0 was used. Data were expressed as mean \pm standard deviation (SD) for continuous variables and percentages (\%) for categorical variables. Student's t-test was used to compare continuous variables. Normality of continuous variables was determined and confirmed using Kolmogorov-Smirnov test. Differences among the groups were analysed using analysis of variance for measurement data, as well as the chi squared test for categorical values. Pearson correlation coefficient and Spearman's correlation coefficient were used to express the correlations between oxLDL/LDL-C ratio and different variables. Multiple or binary logistic regression analysis was performed to evaluate the odds ratio $(\mathrm{OR})$ and associated factors. We calculated the odds ratio $(\mathrm{OR}, 95 \% \mathrm{Cl})$ in two logistic regression models: a non adjusted model and a multivariable model adjusted for all variables, with $p<0.05$ in the non adjusted model. A ROC curve was employed to find the cut off of oxLDL/LDL-C ratio to indicate the occurrence of severe coronary atherosclerosis. All $p$ values were two tailed, and $p<0.05$ was considered to be statistically.

\section{Results}

\section{General data of the patients}

The clinical data of the four groups were collected and compared. There was gradual increase in age form normal group to severe group with statistical difference between severe group and the other three groups. Duration of diabetes has the same trend as age with statistical difference between normal group and severe group. BMI in mild group was the highest among these four groups, and has statistical difference compared with moderate group. There were statistical differences in $\mathrm{HbA} 1 \mathrm{c}$ levels between groups. The lowest LDL-C level was unexpectedly appeared in severe group with statistical difference compared with the other three groups. It is noted that the rate of statin-use in baseline was also the highest in severe group which may be the reason for the lowest LDL-C level. (Table 1)

Table 1. Clinical data of patients. 


\begin{tabular}{|c|c|c|c|c|}
\hline Factor & Normal & Mild Group & Moderate Group & Severe Group \\
\hline Sex (male/female) & $12 / 11$ & $26 / 19$ & $38 / 20$ & $16 / 10$ \\
\hline Age (years) & $58.7 \pm 8.7^{\Delta}$ & $60.3 \pm 6.2^{*}$ & $62.0 \pm 7.2^{*}$ & $65.2 \pm 9.4$ \\
\hline Duration of diabetes (years) & $8.2 \pm 5.4^{*}$ & $9.3 \pm 5.5$ & $10.0 \pm 8.2$ & $12.3 \pm 10.3$ \\
\hline BMI $\left(\mathrm{kg} / \mathrm{m}^{2}\right)$ & $24.7 \pm 3.4$ & $26.2 \pm 4.1^{\#}$ & $24.1 \pm 3.1$ & $24.8 \pm 3.8$ \\
\hline Smoking history (yes/no) & $7 / 16$ & $18 / 27$ & $24 / 34$ & $10 / 16$ \\
\hline HbA1c (\%) & $9.6 \pm 2.6$ & $8.4 \pm 2.2^{\#}$ & $9.8 \pm 2.8$ & $8.4 \pm 1.9^{\#}$ \\
\hline $\mathrm{TC} \square \mathrm{mmol} / \mathrm{L} \square$ & $4.7 \pm 1.5$ & $4.7 \pm 1.3$ & $4.6 \pm 1.3$ & $4.1 \pm 1.4$ \\
\hline TG $\square \mathrm{mmol} / \mathrm{L} \square$ & $1.7 \pm 0.8$ & $2.0 \pm 0.9$ & $1.9 \pm 2.0$ & $2.0 \pm 0.8$ \\
\hline HDL-C $\square \mathrm{mmol} / \mathrm{L} \square$ & $1.4 \pm 0.7$ & $1.3 \pm 0.7$ & $1.2 \pm 0.6$ & $1.2 \pm 0.6$ \\
\hline LDL-C (mmol/L) & $3.1 \pm 1.3^{*}$ & $3.1 \pm 1.1^{*}$ & $3.0 \pm 1.0 *$ & $2.6 \pm 1.1$ \\
\hline LDL-C $\square \mathrm{mg} / \mathrm{dL} \square$ & $121.3 \pm 51.5^{*}$ & $122.4 \pm 43.4^{*}$ & $118.1 \pm 37.5^{*}$ & $98.6 \pm 44.2$ \\
\hline OxLDL (mg/dl) & $10.3 \pm 4.4$ & $10.2 \pm 5.4$ & $9.4 \pm 4.3$ & $10.6 \pm 5.5$ \\
\hline OxLDL/LDL-C & $0.083 \pm 0.040 *$ & $0.086 \pm 0.036 *$ & $0.085 \pm 0.048^{*}$ & $0.111 \pm 0.052$ \\
\hline Statin treatment (yes/no) & $8 / 15^{*}$ & $15 / 30^{*}$ & $17 / 41^{*}$ & $21 / 5$ \\
\hline Rate of statin using & $34.8 \% *$ & $33.3 \% *$ & $29.3 \% *$ & $80.8 \%$ \\
\hline
\end{tabular}

Continuous variables were expressed as mean $\pm \mathrm{SD}$, while categorical variables were expressed as percentages.

${ }^{*} \mathrm{p}<0.05$ vs. severe group; $\Delta \mathrm{p}<0.05$ vs. moderate and severe group; $\# \mathrm{p}<0.05$ vs. moderate group; BMI, body mass index; HbA1c, glycosylated haemoglobin; LDL-C, low-density lipoprotein cholesterol.

\section{Comparison of oxLDL/LDL-C ratio and other interested biomarkers levels in different coronary atherosclerosis groups}

OxLDL/LDL-C ratio in severe group was significantly higher than which in the other three groups respectively $(0.111 \pm 0.052$ vs. $0.083 \pm 0.040,0.086 \pm 0.036,0.085 \pm 0.048$ respectively, $p<0.05)$ (Figure 1).When oxLDL/LDL-C ratio was stratified into two groups according to the median $(\leq 0.0819$ were assigned to the low-ratio group; $>0.0819$ were assigned to the high-ratio group), the Gensini scores were significantly higher in high-ratio group than in low-ratio group $(25.34 \pm 5.72$ vs $12.43 \pm 2.21, \mathrm{P}=0.038)$ (Figure 1). The rate of patients with severe coronary atherosclerosis in high-ratio group was significantly higher than that in low-ratio group, $\mathrm{P}=0.039$ ) (Figure 2).

Fig 1. OxLDL/LDL-C ratio and severity of coronary atherosclerosis in diabetic patients. (A) oxLDL/LDL-C ratio among four groups of different extent of coronary atherosclerosis. ${ }^{*} p<0.05$ compared with severe group. (B) Gensini scores of coronary atherosclerosis between low and high oxLDL/LDL-C ratio groups. ${ }^{*} \mathrm{p}<0.05$ compared with high oxLDL/LDL-C ratio group.

Fig 2. Rate of severe coronary atherosclerosis in low and high oxLDL/LDL-C ratio groups of diabetic patients. ${ }^{*} \mathrm{p}<0.05$ compared with low oxLDL/LDL-C ratio group.

We also found that the patients in severe group had higher VCAM-1 level than those in the other three groups ( $51.32 \pm 23.10$ vs. $36.90 \pm 27.76,41.41 \pm 22.48,44.62 \pm 26.32$ respectively, $p<0.005$ ) (Figure 3 ). There 
were no differences in the level of IL33, FGF21 among these four groups.

Fig 3. VCAM-1 levels among four groups of different extent of coronary atherosclerosis. ${ }^{\star} \mathrm{p}<0.05$ compared with severe group.

\section{Correlation of variables with severity of coronary atherosclerosis in diabetic patients}

Spearman's correlation analysis showed age, and oxLDL/LDL-C ratio were positively correlated with severity of coronary atherosclerosis(both $p<0.05$ ).

Moreover, taking low oxLDL/LDL-C ratio group as the referent, we evaluated the correlation between oxLDL/LDL-C ratio and severity of coronary atherosclerosis in two statistical models by ordinal logistic regression (Table 2). In non-adjusted model, a significant positive correlation was observed between severity of coronary atherosclerosis and high oxLDL/LDL-C ratio (OR 1.83, 95\% Cl 1.21-2.77, $\mathrm{p}<0.01$ ). After adjusted for age, duration, HBA1C and LDL-C level, the positive correlation between severity of coronary atherosclerosis and high oxLDL/LDL-C ratio still existed (OR 2.03, 95\% $\mathrm{Cl} 1.31-3.14, \mathrm{p}<0.01)$.

Table 2 Odds ratio analysis of oxLDL/LDL ratio with severity of coronary atherosclerosis in patients with diabetes

\begin{tabular}{lllll}
\hline \multicolumn{5}{c}{ Severity of coronary atherosclerosis } \\
\hline & Non-adjusted model & \multicolumn{2}{l}{ Multivariate } \\
\hline OxLDLdel*
\end{tabular}

*Variables with $\mathrm{p}<0.05$ in univariate analysis were included in the multivariate model

\section{Assessment of cut-off point of oxLDL/LDL-C ratio for diabetic patients with severe coronary atherosclerosis}

The cut-off value of oxLDL/LDL-C ratio in predicting patients with severe coronary atherosclerosis was derived using ROC curve analysis. The ROC area was 0.651 (95\% $\mathrm{Cl} 0.517-0.785, \mathrm{p}=0.028)$. OxLDL/LDL$C$ ratio identified diabetic patients with severe coronary atherosclerosis with specificity of $68.2 \%$ and sensitivity of $64.9 \%$ and with a cut-off value of $>0.091$. (Figure 4)

Fig 4. ROC curve for oxLDL/LDL-C ratio in prediction by severe coronary atherosclerosis.

\section{Correlation between oxLDL/LDL-C ratio and other variable}

No correlation was found between oxLDL/LDL-C ratio and age, duration of diabetes, use of statin, $\mathrm{HbA1c}$, BMI, LDL-C, FGF21, and VCAM-1 levels. Only IL33 showed positive correlation with oxLDL/LDL-C ratio 
tested by Spearman analysis $(r=0.339, p<0.001)$. When all patients were divided in two groups: low oxLDL/LDL-C ratio group and high oxLDL/LDL-C ratio group, IL33 levels were significantly higher in the high oxLDL/LDL-C ratio group compared with the low group ( $5.34 \pm 0.25$ vs. $5.19 \pm 0.22, p=0.001)$.

We divided the patients into two groups based on whether they had used statins. The results indicated that level of serum LDL-C and oxLDL were both decreased significantly in statin-user compared with nonstatin-user (LDL-C:2.44 $\pm 1.06 \mathrm{mmol} / \mathrm{L}$ vs. $3.38 \pm 0.97 \mathrm{mmol} / \mathrm{L}, \mathrm{P}<0.01 ; \mathrm{oxLDL}: 8.86 \pm 4.58 \mathrm{mg} / \mathrm{dl}$ vs. $10.63 \pm 4.66 \mathrm{mg} / \mathrm{dl}, \mathrm{P}<0.05)$, but oxLDL/LDL-C ratio was not significant changed in statin-user (0.101 \pm 0.051 vs. $0.085 \pm 0.042, P=0.065)$. (Fig 5)

Fig 5. Serum level of LDL-C, oxLDL and oxLDL/LDL-C in statin-using or non-statin-using group. * $\mathrm{p}<0.05$ compared with non-statin-using group.

\section{Comparison of oxLDL/LDL-C ratio and other biomarkers between diabetic patients with and without significant coronary lesions}

Lesions were considered significant if the coronary stenosis was $\geq 50 \%$ diameter and insignificant if the stenosis was $<50 \%$. There were significant differences in oxLDL/LDL-C ratio, IL33 and VCAM- 1 levels between diabetic patients with and without significant coronary lesions $(p<0.05)($ Table 3$)$.

Table 3. Biomarkers in diabetic patients with and without significant coronary lesions.

\begin{tabular}{llll}
\hline Biomarkers & significant & Non-significant & $P$ \\
\hline OxLDL/LDL ratio & $0.108 \pm 0.056$ & $0.081 \pm 0.035$ & 0.005 \\
FGF21 & $0.26 \pm 0.020$ & $0.27 \pm 0.020$ & NS \\
IL33 & $5.29 \pm 0.26$ & $5.24 \pm 0.23$ & 0.047 \\
VCAM-1 & $50.56 \pm 23.60$ & $40.96 \pm 27.01$ & 0.001 \\
\hline
\end{tabular}

Continuous variables were expressed as mean \pm SD.

\section{Discussion}

Although the invasive conventional coronary angiography (ICA) is the golden standard to evaluate the severity of coronary lesions, its use in the clinical arena of T2DM patients is limited. This is an invasive technique that can rarely be used to evaluate the minor or moderate coronary lesions with no obvious clinical symptoms ${ }^{[35]}$. In order to avoid the selection bias toward subjects with severe coronary lesions, we used CCTA instead of ICA in our study on the basis of the evidences for accuracy of CCTA. In this study, wefound that oxLDL/LDL-C ratio observably associated with severity of diabetic coronary lesions and significantly increased in patients with severe coronary lesions. This suggested that oxLDL/LDL-C ratio may be a potential biomarker for screening severe coronary atherosclerosis in patients with T2DM. 
OxLDL plays an important role in atherogenesis by promoting an inflammatory environment and lipid deposition in the arterial wall ${ }^{[36]}$. Therefore, elevated levels of circulating oxLDL or oxLDL/LDL-C ratio have been demonstrated in large prospective cohorts, powered for clinical endpoints, to confer an adverse CVD prognosis. The Bruneck Study showed a significant association between oxidation-specific epitopes biomarkers including oxidized phospholipids of ApoB-100 and the development of adverse CVD events over 15-year follow up ${ }^{[37]}$. A recent meta-analysis supported these findings, reporting that elevated serum oxLDL is associated with an increased risk of CVD events ${ }^{[38]}$. In a previous prospective nested casecontrol study of initially healthy men, plasma oxLDL concentrations were significantly higher in men who subsequently experienced a coronary event than in the matched controls ${ }^{[11]}$.Moreover, oxLDL is significantly associated with diabetes and its complications. There are significant higher levels of oxLDL in patients with diabetes compared with non-diabetics ${ }^{[39]}$. A significant association of plasma oxLDL/LDL-C with CHD was found in males with diabetes in a 10-year prospective study [40]. Most of studies were focus on the association of oxLDL and/or oxLDL/LDL-C with the cardiovascular events. None of studies demonstrated the correlation between oxLDL/LDL-C ratio and the severity of coronary atherosclerosis in patients with diabetes. Faviou $\mathrm{E}$ et al reported that serum oxLDL level was associated with coronary artery disease as well as with the severity of the clinical presentation. However, this study didn't estimate the coronary artery lesions by ICA or CCTA ${ }^{[41]}$. To this extent, our study for the first time demonstrated that oxLDL/LDL-C ratio could reflect the severity of coronary atherosclerosis evaluated by CCTA in patients with diabetes. This biomarker may provide a tool to identify the severe coronary artery lesions earlier in order to prevent cardiovascular events.

As for the influencing factors of severity of coronary lesions, the results of our study showed a positive correlation between severity of coronary lesions and age, as well as no correlation with duration of diabetes, HbA1c and LDL-C levels. When divided all patients into four groups according to the Gensini scores, the severe group had the longest duration of diabetes and lowest LDL-C levels which may be caused by the highest rate of statin-use. Our study indicated that statins-use decreased both LDL-C and oxLDL levels, however, oxLDL/LDL-C ratio was not affected.

More importantly, this study demonstrated the predicting and screening value of oxLDL/LDL-C ratio for severe coronary atherosclerosis in patients with type 2 diabetes. The present study showed a positive correlation between severity of coronary lesions and oxLDL/LDL-C ratio even adjusted for age, duration and other associated variables. The risk for developing more severe coronary atherosclerosis in high oxLDL/LDL-C ratio group was nearly two-times higher than low oxLDL/LDL-C ratio group. After dividing all patients with T2DM into four groups from normal to severe according to Gensini scores, we found that there was the highest oxLDL/LDL-C ratio in severe group which had a significant difference compared with other three groups. Gensini scores presenting the severity of coronary lesions were in the high oxLDL/LDL-C ratio group than which in the low group. The cut-off for oxLDL/LDL-C ratio to discriminate severe coronary atherosclerosis from normal to moderate lesions was 0.091 for oxLDL/LDL-C ratio, yielding acceptable sensitivity and specificity for predicting severe coronary atherosclerosis in this study. 
Though hyperglycemia can induce oxidative stress and modify LDL-C into pro-atherogenic oxLDL [8] [42] There was no correlation between oxLDL/LDL-C ratio and levels in HbA1c or LDL-C in our study which was conflict with the previous report ${ }^{[43]}$. This discrepancy in the results could be due to the poorly glycemic control and the effect of statin therapy at baseline. It is noted that with the lowest LDL-C levels and highest rate of statin-use, severe group still had the highest oxLDL/LDL-C ratio. These results indicated that statin treatment may be useless in decreasing circulating oxLDL/LDL-C ratio. Studies investigating association between circulating oxLDL and the statin treatment yielded conflicting results. In several studies, the levels of circulating oxLDL were significantly decreased by treatment with statins in patients with type 2 diabetes ${ }^{[44]}$ and $\mathrm{CHD}^{[45]}$. In contrast, Kudret Keskin et al showed that statin treatment had no effect on serum oxLDL level ${ }^{[46]}$.Our study provided new evidence to provethat statin treatment can decrease both LDL-C and oxLDL levels.

Besides oxLDL/LDL-C ratio, other interested biomarkers such as FGF21, IL33 and VCAM-1 which were reported to be associated with diabetes or CAD were also evaluated in the present study. None of them were indicated to be correlated with the severity of coronary atherosclerosis in patients with T2DM. Only VCAM-1 had the similar trend with oxLDL/LDL-C ratio that had the highest levels in the patients of severe group with statistical significance. As a classical cell adhesion molecules, VCAM-1 combined with oxLDL/LDL-C ratio may be more effective than oxLDL/LDL-C ratio alone to predict and screen severe coronary atherosclerosis. IL33 was positively correlated with oxLDL/LDL-C ratio. Moreover, IL33 levels were higher in the high oxLDL/LDL-C ratio group than in the low group. Previous study had demonstrated that oxLDL can stimulated human umbilical vein endothelial cells and vascular smooth muscle cells to release large amounts of thymic stromal lymphopoietin (TSLP). TSLP-IL33 signaling pathway might interact with each other in Th2 cell-mediated inflammatory responses to play its athero-protective role ${ }^{[47]}$. It may be the explanation for the correlation between oxLDL/LDL-C ratio and IL33. We proposed that IL33 may be an alarmin of oxLDL/LDL-C ratio in patients with T2DM. We also found that all of the three biomarkers including oxLDL/LDL-C ratio, IL33 and VCAM-1 were higher in the diabetic patients with significant coronary lesions than in the patients with non-significant lesions. These results further supported the value of these three biomarkers in predicting and screening severe coronary atherosclerosis in patients with T2DM.

Some limitations of this study should also be considered. Firstly, the number of the samples was relatively small and recruited patients were all inpatients with poor glycemic control. Finally, it was a cross-sectional study, lacking the long-term outcome of coronary atherosclerosis and oxLDL/LDL-C ratio changing in patients with T2DM. It needs further follow-up study to identify the detailed association between the oxLDL/LDL-C ratio and the development of coronary atherosclerosis in type 2 diabetes population.

\section{Conclusion}


In conclusion, our study demonstrated for the first time that oxLDL/LDL-C ratio is significantly associated with the severity of coronary atherosclerosis and predict severe coronary lesions in patients with type 2 diabetes. It may be more effective to test the combination of oxLDL/LDL-C ratio, VCAM-1 and IL33. OxLDL/LDL-C ratio is considered as a potential biomarker in diabetic patients for early recognition and intervention of severe coronary atherosclerosis.

\section{Abbreviations}

ASCVD

Atherosclerosis cardiovascular diseases

OxLDL

Oxidized low-density lipoprotein

LDL-C

Low-density lipoprotein cholesterol

CCTA

Coronary computed tomographic angiography

ROC

receiver operator characteristic

CVD

Cardiovascular diseases

TC

Total cholesterol

HDL-C

High density lipoprotein-cholesterol

Apo

Apolipoprotein

CAD

Coronary artery disease

T2DM

Type 2 diabetes mellitus

CHD

Coronary heart disease

FGF21

Fibroblast growth factor 21

IL33

Interleukin 33

VCAM-1

Vascular cell adhesion molecule-1

ST2

Suppression of tumorigenity 
BMI

Body mass index

TG

Triglycerides

LAD

Left anterior descending artery

SD

Standard deviation

OR

Odds ratio

ICA

Invasive conventional coronary angiography

TSLP

Thymic stromal lymphopoietin.

\section{Declarations}

\section{Ethics approval and consent to participate}

The study protocol was approved by the Human Research and Ethics Committee of Shanghai East Hospital, and the study was conducted according to the principles of the Helsinki Declaration II. All patients provided written informed consent.

\section{Consent for publication}

Not applicable.

\section{Availability of data and materials}

The datasets used and analyzed during the current study are available from the corresponding author on reasonable request.

\section{Competing interests}

The authors declare that they have no competing interests.

\section{Funding}

This work was supported by the National Natural Science Foundation of China (81870529).

\section{Authors' contributions}

$\mathrm{LX}$ and BF contributed to the study design, data acquisition, and data analysis and wrote the manuscript. $\mathrm{XY}$ and $\mathrm{ZT}$ contributed to the data acquisition. All authors gave final approval for this version to be 
published. All authors read and approved the final manuscript.

\section{Acknowledgement}

Not applicable..

\section{References}

1. Wang L, Gao P, Zhang M, Huang Z, Zhang D, Deng Q, et al. Prevalence and Ethnic Pattern of Diabetes and Prediabetes in China in 2013. JAMA, 2017, 317:2515-23.

2. Grundy SM, Benjamin IJ, Burke GL, Chait A, Eckel RH, Howard BV, et al. Diabetes and cardiovascular disease: a statement for healthcare professionals from the American Heart Association. Circulation. 1999;100:1134-46.

3. Hurst RT, Lee RW. Increased incidence of coronary atherosclerosis in type 2 diabetes mellitus: mechanisms and management. Ann Intern Med. 2003;139:824-34.

4. Sandhu PK, Musaad SM, Remaley AT, Buehler SS, Strider S, Derzon JH, et al. Lipoprotein Biomarkers and Risk of Cardiovascular Disease: A Laboratory Medicine Best Practices (LMBP) Systematic Review. J Appl Lab Med. 2016;1:214-29.

5. Yoshida H, Kisugi R. Mechanisms of LDL-C oxidation. Clin Chim Acta. 2010;411:1875-82.

6. Morel DW, Hessler JR, Chisolm GM. Low density lipoprotein cytotoxicity induced by free radical peroxidation of lipid. J Lipid Res. 1983;24:1070-6.

7. Lopez LR, Hurley BL, Simpson DF, Matsuura E. Oxidized low-density lipoprotein/beta2-glycoprotein I complexes and autoantibodies in patients with type 2 diabetes mellitus. Ann N Y Acad Sci. 2005;1051:97-103.

8. Evans JL, Goldfine ID, Maddux BA, Grodsky GM. Oxidative stress and stress-activated signaling pathways: a unifying hypothesis of type 2 diabetes. Endocr Rev. 2002;23:599-622.

9. Liem AH, van de Woestijne AP, Roeters van Lennep HW, Zwinderman AH, van der Steeg WA, Jukema JW. ApoB/A1 and LDL-C/HDL-C and the prediction of cardiovascular risk in statin-treated patients. Curr Med Res Opin. 2008;24:359-64.

10. Wallenfeldt K, Fagerberg B, Wikstrand J, Hulthe J. Oxidized low-density lipoprotein in plasma is a prognostic marker of subclinical atherosclerosis development in clinically healthy men. $\mathrm{J}$ Intern Med. 2004;256:413-20.

11. Meisinger C, Baumert J, Khuseyinova N, Loewel H, Koenig W. Plasma oxidized low-density lipoprotein, a strong predictor for acute coronary heart disease events in apparently healthy, middleaged men from the general population. Circulation. 2005;112:651-7.

12. Huang H, Mai W, Liu D, Hao Y, Tao J, Dong Y. The oxidation ratio of LDL-C: A Predictor for Coronary Artery Disease. Dis Markers. 2008;24:341-9.

13. Johnston N, Jernberg T, Lagerqvist B, Siegbahn A, Wallentin L. Oxidized low-density lipoprotein as a predictor of outcome in patients with unstable coronary artery disease. Int J Cardiol. 2006;113:167- 
73.

14. Njajou OT, Kanaya AM, Holvoet P, Connelly S, Strotmeyer ES, Harris TB, et al. Association between oxidized LDL-C, obesity and type 2 diabetes in a population-based cohort, the Health, Aging and Body Composition Study. Diabetes/Metabolism Research Reviews. 2009;25:733-9.

15. Huang H, Ma R, Liu D, Liu C, Ma Y, Mai W, et al. Oxidized low-density lipoprotein cholesterol and the ratio in the diagnosis and evaluation of therapeutic effect in patients with coronary artery disease. Dis Markers. 2012;33:295-302.

16. Johnston N, Jernberg T, Lagerqvist B, Siegbahn A, Wallentin L. Improved identification of patients with coronary artery disease by the use of new lipid and lipoprotein biomarkers. Am J Cardiol. 2006;97:640-5.

17. Lankin V, Viigimaa M, Tikhaze A, Kumskova E, Konovalova G, Abina J, et al. Cholesterol-rich low density lipoproteins are also more oxidized. Mol Cell Biochem. 2011;355:187-91.

18. Segev A, Strauss BH, Witztum JL, Lau HK, Tsimikas S. Relationship of a comprehensive panel of plasma oxidized low-density lipoprotein markers to angiographic restenosis in patients undergoing percutaneous coronary intervention for stable angina. Am Heart J. 2005;150:1007-14.

19. Imazu M, Ono K, Tadehara F, Kajiwara K, Yamamoto H, Sumii K, et al. Plasma levels of oxidized low density lipoprotein are associated with stable angina pectoris and modalities of acute coronary syndrome. Int Heart J. 2008;49:515-24.

20. Tsimikas S, Brilakis ES, Miller ER, McConnell JP, Lennon RJ, Kornman KS, et al. Oxidized phospholipids, Lp(a) lipoprotein, and coronary artery disease. N Engl J Med. 2005;353:46-57.

21. Ndrepepa G, Tada T, Fusaro M, Cassese S, King L, Hadamitzky M, et al. Association of coronary atherosclerotic burden with clinical presentation and prognosis in patients with stable and unstable coronary artery disease. Clin Res Cardiol. 2012;101(12):1003-11.

22. Larson DB, Johnson LW, Schnell BM, Salisbury SR, Forman HP. National trends in CT use in the emergency department: 1995-2007. Radiology. 2011;258:164-73.

23. Hulten E, Villines TC, Cheezum MK, Berman DS, Dunning A, Achenbach S, et al. CONFIRM Investigators. Usefulness of coronary computed tomography angiography to predict mortality and myocardial infarction among Caucasian, African and East Asian ethnicities (from the CONFIRM [Coronary CT Angiography Evaluation for Clinical Outcomes: An International Multicenter] Registry). Am J Cardiol. 2013;111:479-85.

24. Papadopoulou SL, Girasis C, Dharampal A, Farooq V, Onuma Y, Rossi A, et al. CT-SYNTAX score: a feasibility and reproducibility Study. JACC Cardiovasc Imaging. 2013;6:413-5.

25. Chow BJ, Small G, Yam Y, Chen L, Achenbach S, Al-Mallah M, et al. CONFIRM Investigators. Incremental prognostic value of cardiac computed tomography in coronary artery disease using CONFIRM: Coronary computed tomography angiography evaluation for clinical outcomes: an InteRnational Multicenter registry. Circ Cardiovasc Imaging. 2011;4:463-72.

26. Roos CJ, Kharagjitsingh AV, Jukema JW, Bax JJ, Scholte AJ. Comparison by computed tomographic angiography-the presence and extent of coronary arterial atherosclerosis in South Asians versus 
Caucasians with diabetes mellitus. Am J Cardiol. 2014;113:1782-7.

27. Farrag A, Bakhoum S, Salem MA, El-Faramawy A, Gergis E. The association between extracoronary calcification and coronary artery disease in patients with type 2 diabetes mellitus. Heart Vessels. 2013;28:12-8.

28. Fisher FM, Maratos-Flier E. Understanding the Physiology of FGF21. Annu Rev Physiol. 2016;78:22341.

29. Zhang X, Yeung DC, Karpisek M, Stejskal D, Zhou ZG, Liu F, et al. Serum FGF21 levels are increased in obesity and are independently associated with the metabolic syndrome in humans. Diabetes. 2008;57:1246-53.

30. Wu L, Qian L, Zhang L, Zhang J, Zhou J, Li Y, et al. Fibroblast Growth Factor 21 is Related to Atherosclerosis Independent of Nonalcoholic Fatty Liver Disease and Predicts Atherosclerotic Cardiovascular Events. J Am Heart Assoc. 2020;9:e015226.

31. Schmitz J, Owyang A, Oldham E, Song Y, Murphy E, McClanahan TK, et al. IL-33, an interleukin-1-like cytokine that signals via the IL-1 receptor-related protein ST2 and induces T helper type 2-associated cytokines. Immunity. 2005;23:479-90.

32. Marzullo A, Ambrosi F, Inchingolo M, Manca F, Devito F, Angiletta D, et al. ST2L Transmembrane Receptor Expression: An Immunochemical Study on Endarterectomy Samples. PLoS One. 2016;11(5):e0156315.

33. Koh Y, Park J. Cell adhesion molecules and exercise. J Inflamm Res. 2018;11:297-306.

34. Gensini GG. A more meaningful scoring system for determining the severity of coronary heart disease. Am J Cardiol. 1983;51:606.

35. Meijboom WB, Meijs MF, Schuijf JD, Cramer MJ, Mollet NR, van Mieghem CA, et al. Diagnostic accuracy of 64-slice computed tomography coronary angiography: a prospective, multicenter, multivendor study. J Am Coll Cardiol. 2008;52:2135-44.

36. Goyal T, Mitra S, Khaidakov M, Wang X, Singla S, Ding Z, et al. Current Concepts of the Role of Oxidized LDL Receptors in Atherosclerosis. Curr Atheroscler Rep. 2012 Jan 29. doi:10.1007/s11883012-0228-1.

37. Tsimikas S, Willeit P, Willeit J, Santer P, Mayr M, Xu Q, et al. Oxidation-specific biomarkers, prospective 15-year cardiovascular and stroke outcomes, and net reclassification of cardiovascular events. J Am Coll Cardiol. 2012;60:2218-29.

38. Gao S, Zhao D, Wang M, Zhao F, Han X, Qi Y, et al. Association between circulating oxidized LDL-C and atherosclerotic cardiovascular disease: a meta-analysis of observational studies. Can J Cardiol. 2017;33:1624-32.

39. Hoogeveen RC, Ballantyne CM, Bang H, Heiss G, Duncan BB, Folsom AR, et al. Circulating oxidised low-density lipoprotein and intercellular adhesion molecule-1 and risk of type 2 diabetes mellitus: the Atherosclerosis Risk in Communities Study. Diabetologia. 2007;50:36-42.

40. Stephens JW, Gable DR, Hurel SJ, Miller GJ, Cooper JA, Humphries SE. Increased plasma markers of oxidative stress are associated with coronary heart disease in males with diabetes mellitus and with 
10-year risk in a prospective sample of males. Clin Chem. 2006;5:446-52.

41. Faviou E, Vourli G, Nounopoulos C, Zachari A, Dionyssiou-Asteriou A. Circulating oxidized low density lipoprotein, autoantibodies against them and homocysteine serum levels in diagnosis and estimation of severity of coronary artery disease. Free Radic Res. 2005;39:419-29.

42. Laakso M. Hyperglycemia and cardiovascular disease in type 2 diabetes. Diabetes. 1999;48:937-42.

43. Harmon ME, Campen MJ, Miller C, Shuey C, Cajero M, Lucas S, et al. Associations of Circulating Oxidized LDL and Conventional Biomarkers of Cardiovascular Disease in a Cross-Sectional Study of the Navajo Population. PLoS One. 2016;11:e0143102.

44. Akalin A, Temiz G, Akcar N, Sensoy B. Short term effects of atorvastatin on endothelial functions and oxidized LDL-C levels in patients with type 2 diabetes. Endocr J. 2008;55:861-6.

45. Tavridou A, Efthimiadis A, Efthimiadis I, Paschalidou H. Antioxidant effects of simvastatin in primary and secondary prevention of coronary heart disease. Eur J Clin Pharmacol. 2006;62:485-9.

46. Keskin K, Kilci H, Aksan G, Çetinkal G, Yıldız SS, Kocaman Türk F, et al. Serum oxidized low-density lipoprotein level as a marker of oxidative stress in patients undergoing hyperbaric oxygen therapy. Turk Kardiyol Dern Ars. 2017;45:533-7.

47. Nie SF, Zha LF, Fan Q, Liao YH, Zhang HS, Chen QW, et al. Genetic Regulation of the Thymic Stromal Lymphopoietin (TSLP)/TSLP Receptor (TSLPR) Gene Expression and Influence of Epistatic Interactions Between IL-33 and the TSLP/TSLPR Axis on Risk of Coronary Artery Disease. Front Immunol. 2018;9:1775.

\section{Figures}



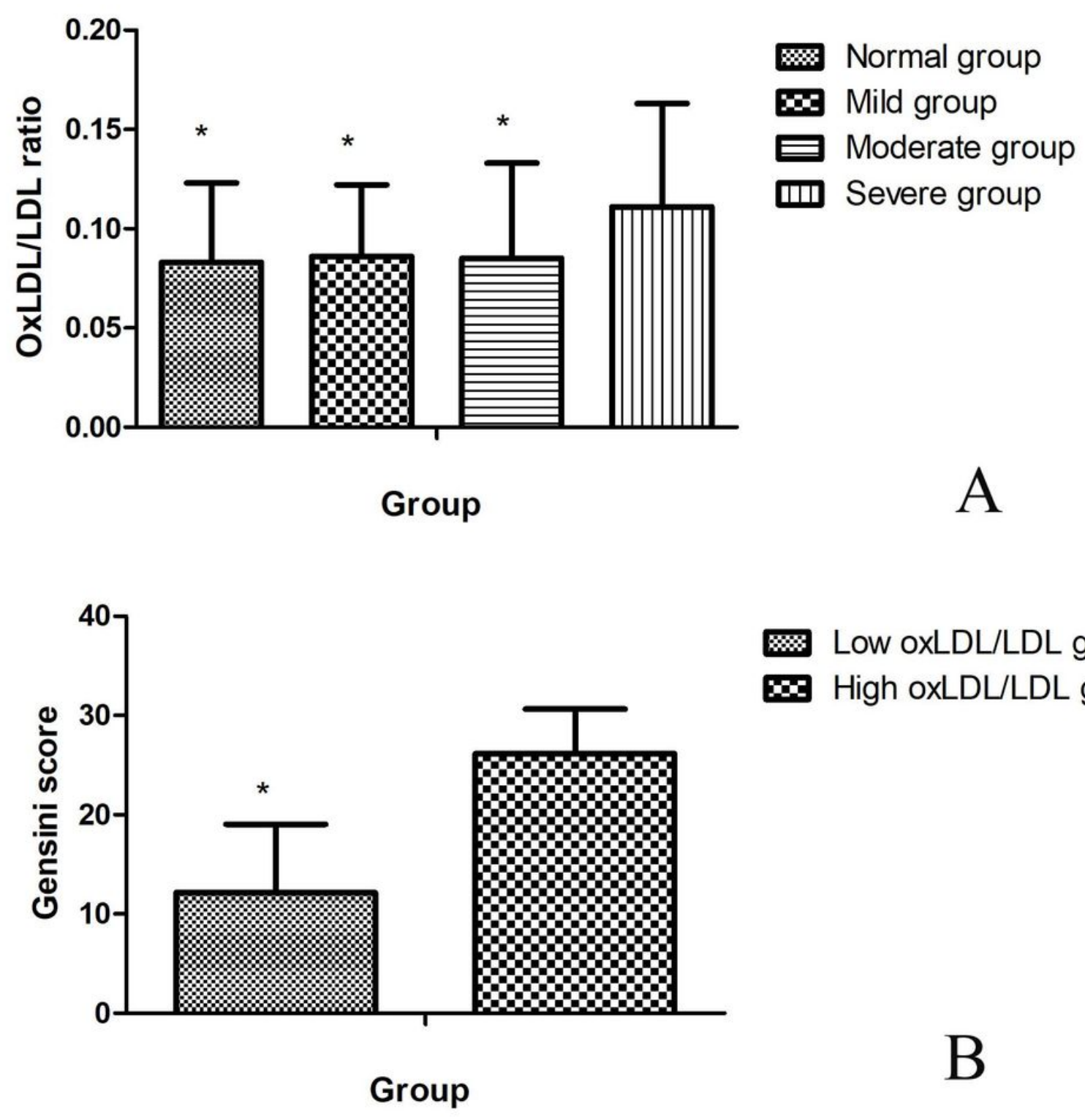

Low oxLDL/LDL group High oxLDL/LDL group

B

Figure 1

OxLDL/LDL-C ratio and severity of coronary atherosclerosis in diabetic patients. (A) oxLDL/LDL-C ratio among four groups of different extent of coronary atherosclerosis. ${ }^{*} p<0.05$ compared with severe group. (B) Gensini scores of coronary atherosclerosis between low and high oxLDL/LDL-C ratio groups. ${ }^{*}<0.05$ compared with high oxLDL/LDL-C ratio group. 


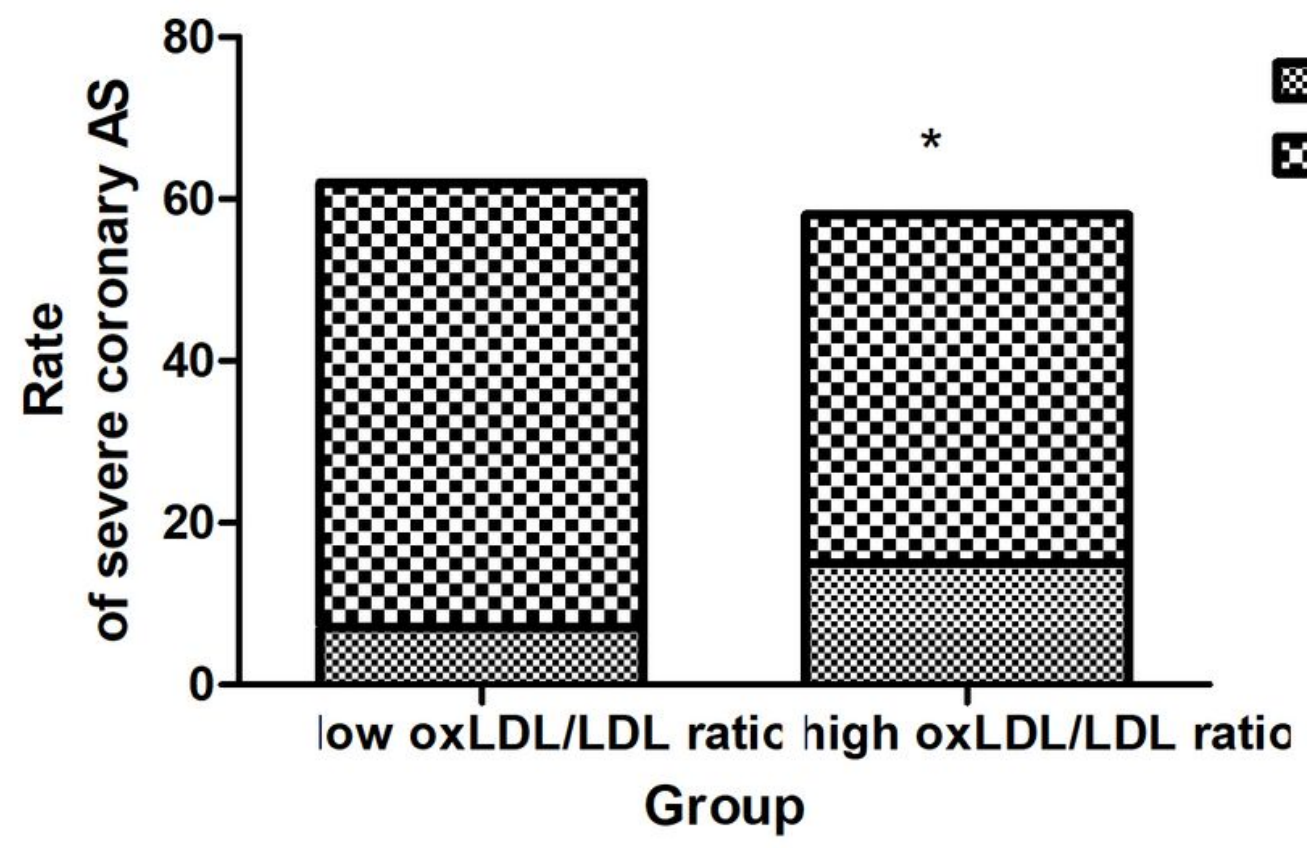

Figure 2

Rate of severe coronary atherosclerosis in low and high oxLDL/LDL-C ratio groups of diabetic patients. ${ }^{*} \mathrm{p}<0.05$ compared with low oxLDL/LDL-C ratio group.

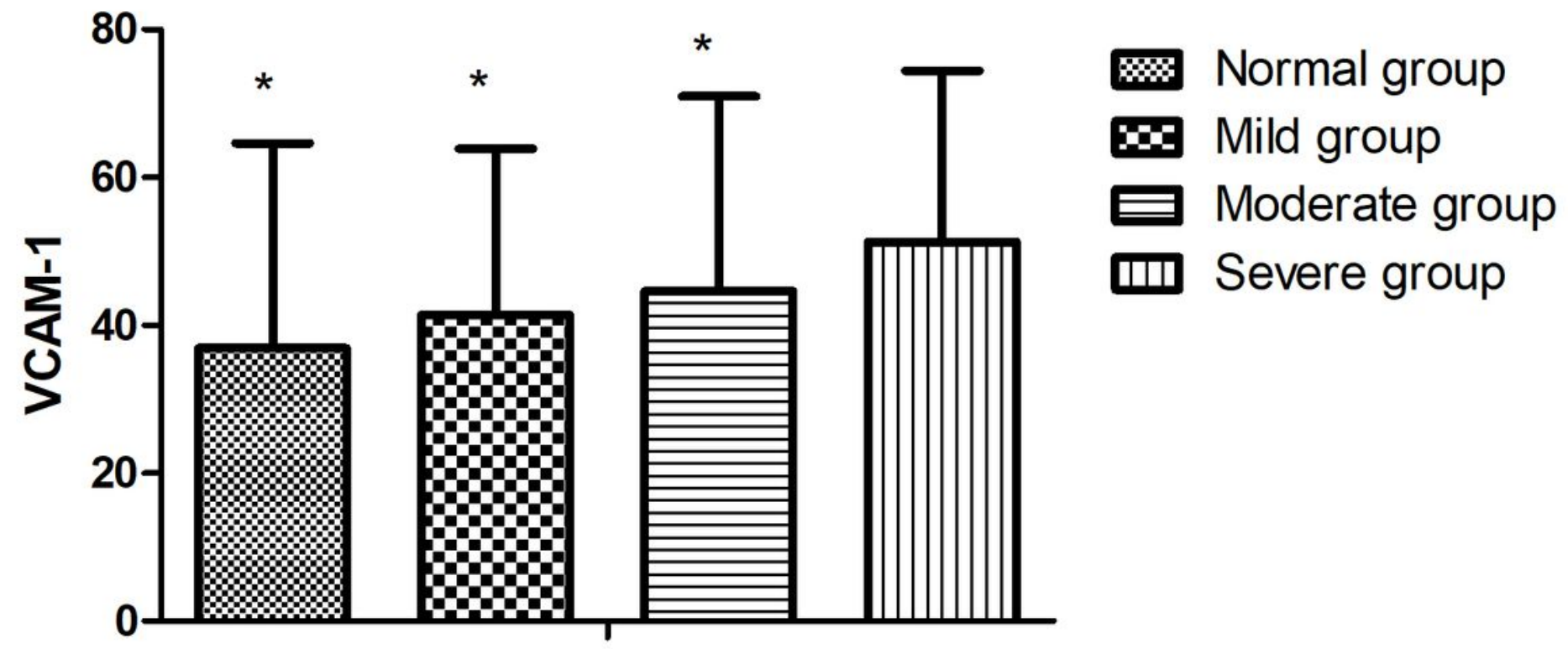

Group

Figure 3 
VCAM- 1 levels among four groups of different extent of coronary atherosclerosis. ${ }^{*} p<0.05$ compared with severe group.

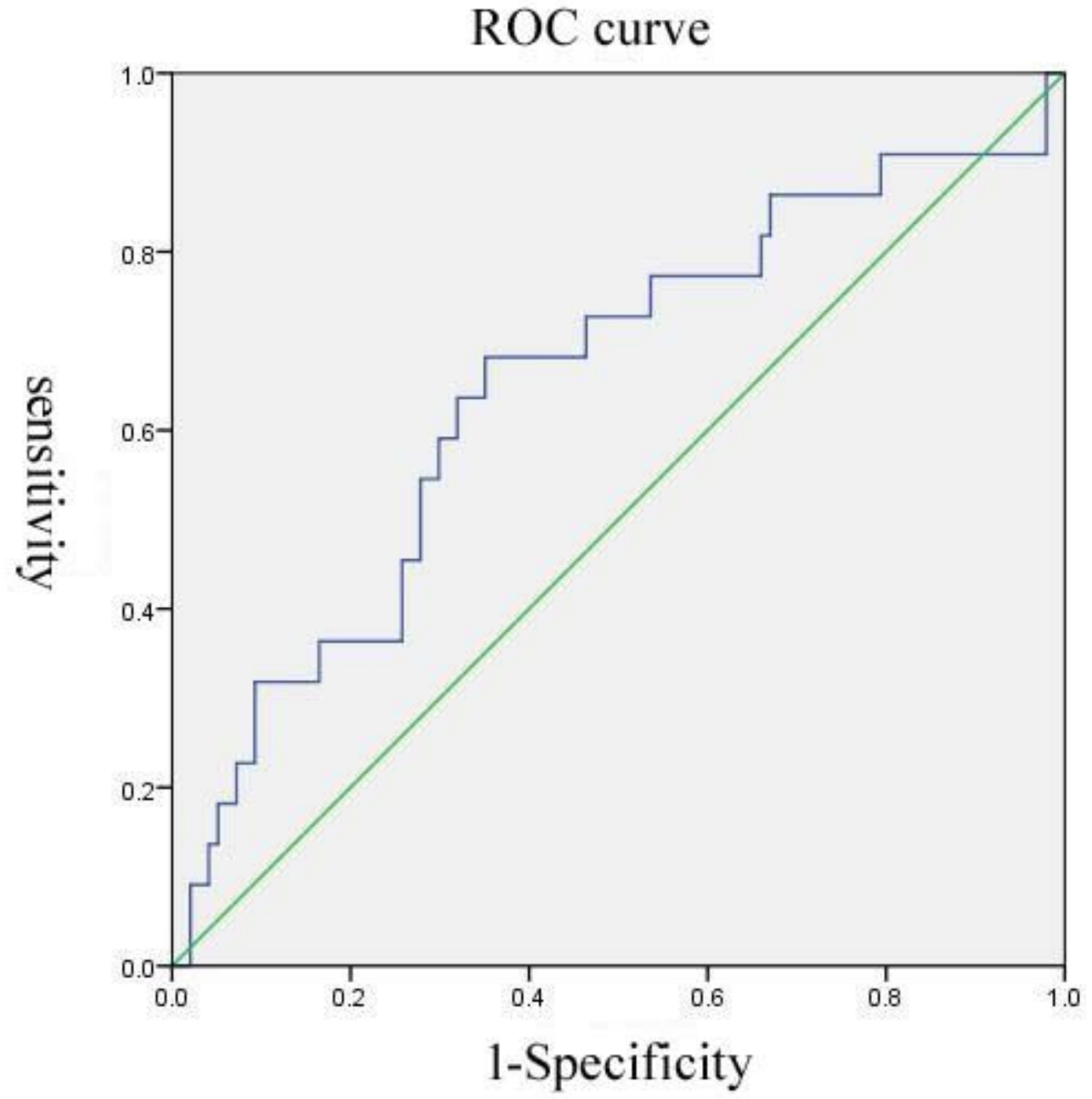

Figure 4

ROC curve for oxLDL/LDL-C ratio in prediction by severe coronary atherosclerosis. 


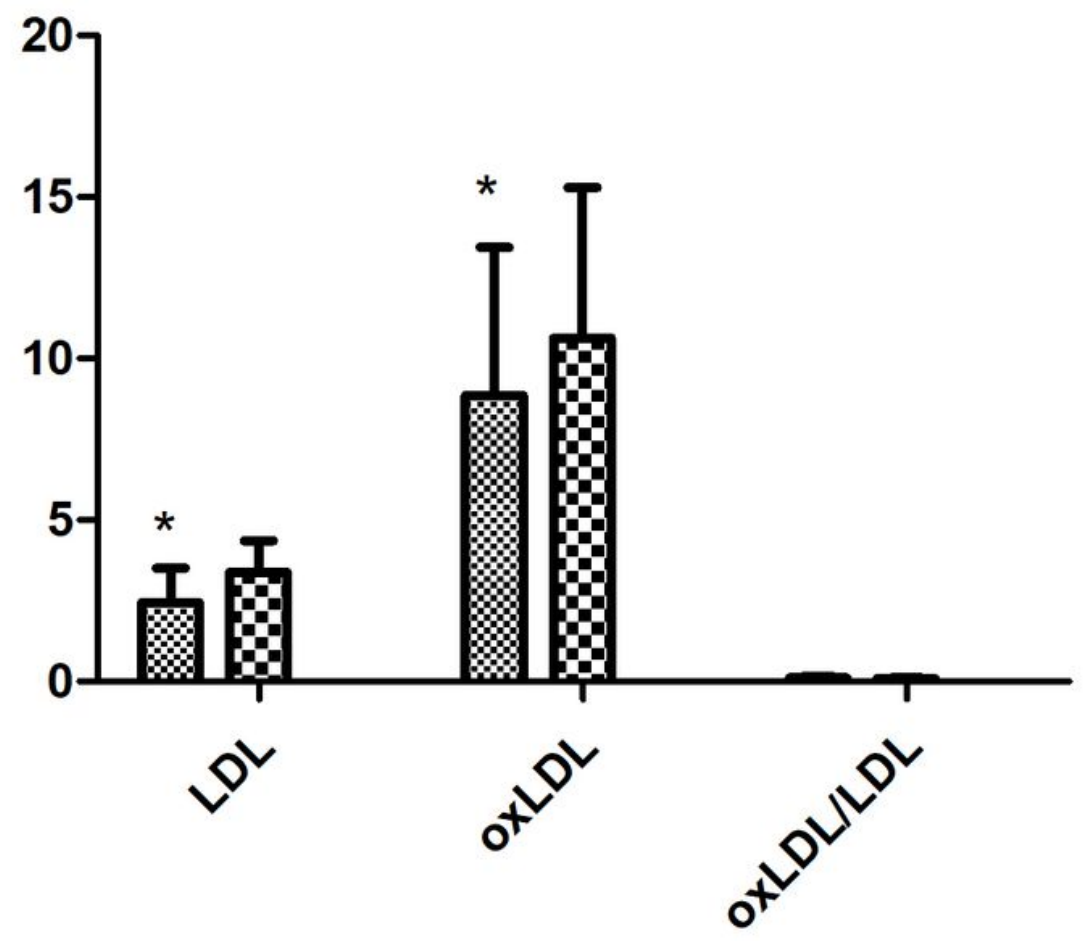

m statin-using group $\mathbf{m o n - s t a t i n - u s i n g ~ g r o u p ~}$

Figure 5

Serum level of LDL-C, oxLDL and oxLDL/LDL-C in statin-using or non-statin-using group. * $\mathrm{p}<0.05$ compared with non-statin-using group. 